\title{
Compromiso de conciencia y bradicardia
}

\author{
Alejandro Paredes, Alex Bittner, Ismael Vergara. \\ Unidad de Electrofisiología y Arritmología Cardiaca, División de Enfermedades Cardiovasculares - \\ Pontificia Universidad Católica de Chile.
}

Mujer de 71 años, institucionalizada con antecedentes de esquizofrenia y tabaquismo. Consulta en el servicio de urgencias tras ser encontrada a la intemperie comprometida de conciencia. Al llegar la ambulancia se constata mal perfundida, bradipsíquica y bradicárdica, siendo trasladada al servicio de Urgencia. A su ingreso el ECG mostró bradicardia sinusal con trastorno de la conducción intraventricular y prolongación del intervalo QT. Los exámenes de laboratorio al ingreso resultaron dentro de límites normales. La historia clínica y los trazados electrocardiográficos son presentados, siendo discutidos junto al manejo médico.

\section{Unconciousness and bradycardia}

A 71year old woman, institutionalized with a history of schizophrenia and smoking. She was transported to a local emergency room after being found laying outside unconscious. She was hypoperfused, bradypsychic and bradycardic, being transferred to the emergency servi- ce. On admission, the ECG showed sinus bradycardia with intraventricular conduction delay and QT prolongation. Laboratory tests were normal. Clinical history, physical examination and ECG tracings are presented and management is discussed.. 


\section{Caso clínico}

Se presenta el caso clínico de una paciente de sexo femenino, de 71 años institucionalizada con antecedentes de esquizofrenia (diagnosticada a los 18 años) y tabaquismo severo. Consulta en el servicio de urgencias tras ser encontrada a la intemperie comprometida de conciencia. Al llegar la ambulancia se constata mal perfundida, bradipsíquica y bradicárdica, siendo trasladada al servicio de Urgencia. A su ingreso el ECG mostró bradicardia sinusal $43 \mathrm{lpm}$ con trastorno de la conducción intraventricular y prolongación del intervalo QT (Figura 1). Sin historia previa de síncope. Al examen físico destaca PA $85 / 57 \mathrm{~mm}$ $\mathrm{Hg}$, enflaquecida, mala perfusión distal con lleve capilar enlentecido y disminución global del murmullo pulmonar. Los exámenes de laboratorio solicitados al ingreso fueron: potasio $4.2 \mathrm{mEq} / \mathrm{L}$, calcio $9.9 \mathrm{mg} / \mathrm{dL}$. Hemograma y función renal dentro de límites normales. TAC de cerebro mostró leve hidrocefalia sin otros hallazgos de significado patológico.

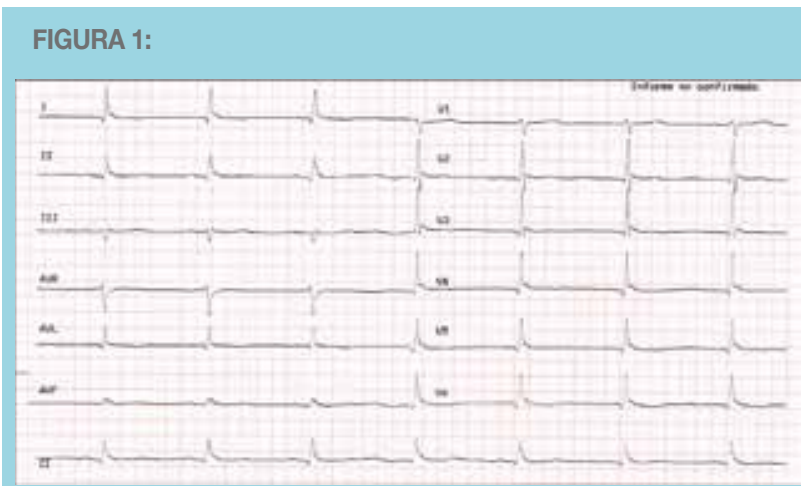

ECG DE INGRESO AL SERVICIO DE URGENCIA.
Al ingreso a dicha unidad se constata paciente con temperatura corporal central de $28^{\circ} \mathrm{C}$. Del ECG destaca trastorno de la conducción intraventricular y presencia de una deflexión al final del complejo QRS que corresponde a la onda J o de Osborn, además de prolongación de los intervalos QRS y QT, hallazgos que se explicarían en el contexto de hipotermia. Se realiza recalentamiento activo con medidas físicas y endovascular con el objetivo de elevar su temperatura en $0.5^{\circ} \mathrm{C} / \mathrm{hr}$, logrando normotermia a las $12 \mathrm{hr}$ desde el ingreso. La electrocardiografía mostró normalización del ECG y desaparición de los hallazgos mencionados a las 24-48 hr de lograda la normalización de la temperatura corporal (Figuras 2 y 3). La conclusión de la evaluación neurológica y psiquiátrica fue que el compromiso de conciencia se explicaría por ajuste reciente de psicofármacos. La paciente evolucionó satisfactoriamente, y su ECG de alta mostró ritmo sinusal a 75 latidos por min.

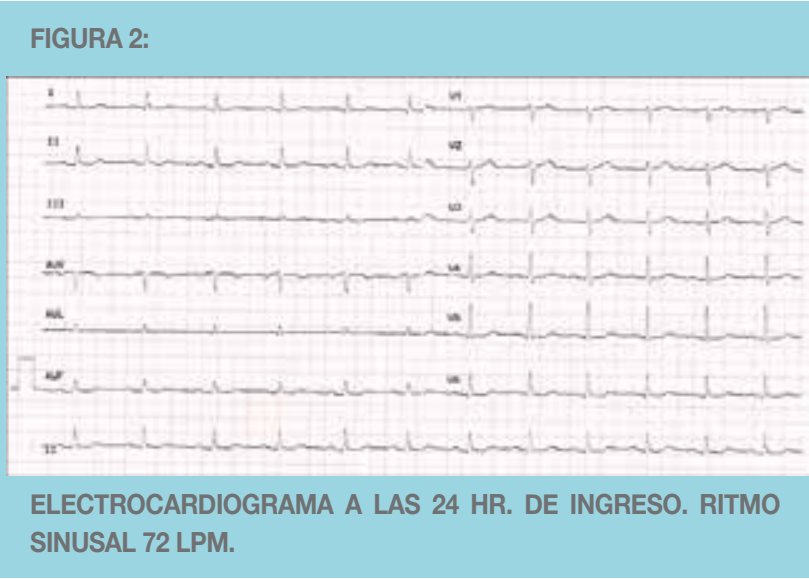

Se hospitaliza en Unidad de cuidados intensivos para manejo, solicitando evaluación cardiológica por bradicardia y compromiso de conciencia asociado.

Por favor analice el ECG en el contexto de la historia de la paciente. Posteriormente, refiérase a la columna siguiente para la discusión del caso.

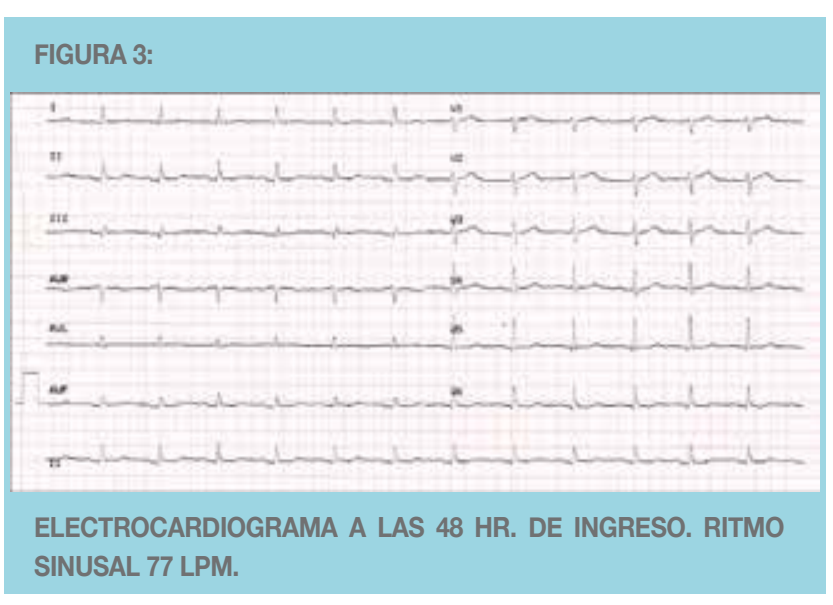




\section{Discusión}

La Hipotermia se define como una temperatura corporal central inferior a $35^{\circ} \mathrm{C}$ y se clasifica en primaria (accidental) o secundaria. Dependiendo de su magnitud, se puede subdividir en leve $\left(32-35^{\circ} \mathrm{C}\right)$, moderada $\left(28-32^{\circ} \mathrm{C}\right)$, severa $\left(<28^{\circ} \mathrm{C}\right)$ y profunda $\left(<24^{\circ} \mathrm{C}\right)$

\section{Cambios electrocardiográficos observados en hipotermia:}

- Artefactos por calofríos.

- Fibrilación auricular con respuesta ventricular lenta.

- Ondas J o de Osborn.

- Bradicardia, especialmente con ritmos de la unión.

- Prolongación de los intervalos PR, QRS y QT.

- Elevación o depresión del segmento ST.

- Arritmias ventriculares: extrasistolia, taquicardia ventricular o fibrilación. - Asistolia.

Existen diversas manifestaciones electrocardiográficas de la hipotermia (Tabla 1). Las más comunes incluyen artefactos por calofríos y bradicardia. Frecuentemente, se asocia a la aparición de ondas $\mathbf{J}$ (también llamada onda de Osborn, descrita por primera vez el año $1953^{1}$ ) y a la prolongación de los intervalos PR, QRS, y QT). También pueden presentarse alteraciones en la repolarización ventricular (elevación o depresión del segmento ST) que pue- den imitar a un síndrome coronario agudo.

Tabla 1: Cambios electrocardiográficos en hipotermia.

Tabla 1: Cambios electrocardiográficos en hipotermia.

Fisiopatológicamente, la hipotermia induce un incremento en la actividad transmembrana de las corrientes transitorias de salida de potasio (Ito) las cuales son más prominentes en el epicardio que en el endocardio. Esta distribución heterogénea de las corrientes de potasio, explicarían la aparición de las Ondas $\mathrm{J}^{2}$ que son típicamente observadas en la pared inferior y en las precordiales laterales. Estas corresponden a la presencia de una deflexión "lenta" positiva entre el final del complejo QRS y la primera porción del segmento ST. Está onda se encuentra presente en el $80 \%$ de los pacientes con temperatura corporal menor a $32^{\circ} \mathrm{C}$ y en el $100 \%$ de aquellos con menos de $30.5^{\circ} \mathrm{C}^{3}$. La magnitud de ellas, generalmente, se correlaciona con el grado de hipotermia, aunque hay reportes de la persistencia de estos cambios en el ECG a pesar de haber alcanzado la normotermia.

Su aparición, también puede ocurrir en otras situaciones clínicas como la hipercalcemia, traumatismos craneoencefálicos, hemorragia subaracnoidea, angina vasoespástica, FV idiopática, drogas antiarrítmicas (ej: procainamida y ajmalina), síndrome de Brugada y en la repolarización precoz 4 .

\section{Referencias}

1.- OSBORNE JJ. Experimental hypothermia: respiratory and blood $\mathrm{pH}$ changes in relation to cardiac function. Am J Physiol 1953; 175: 389-98.

2.- ALHADDAD I, KHALIL M, BROWN E. OSBORN WAVES of Hypothermia. Circulation. 2000; 101: e233-e244.
3.- DOSHI HH, GIUDICI MC. The EKG in hypothermia and hyperthermia. J Electrocardiol 2015; 48: 203-9.

4.- SLOVIS C, JENKINS R. ABC of clinical electrocardiography: Conditions not primarily affecting the heart. BMJ. 2002; 324:1320-3. 